\title{
Analysis of Fiberglass Winding Angle on Natural Frequency of Free Vibration of Cylindrical Shell with Asymmetric Boundary Conditions
}

\author{
Zhiwei Wang ${ }^{1, a}$, Bo $\mathrm{Wu}^{1}$, Yanfu Wang ${ }^{1}$ and S. M. Bosiakov ${ }^{2}$ \\ ${ }^{1}$ School of Mechanical and Power Engineering, Harbin University of Science and Technology, \\ Harbin, China 150080 \\ ${ }^{2}$ Faculty of mechanics-mathematics, Belarusian State University, Minsk, Belarusian 220000 \\ awangzhiweihit@126.com
}

Keywords: Anisotropic circular cylindrical shells; GFRP; Winding angle; Natural frequencies; Free vibration ; Energy method.

\begin{abstract}
In order to obtain approximate solution of natural frequencies for the free vibration of anisotropic circular cylindrical shells made of GFRP (glass fiber-reinforced plastic) with asymmetric boundary conditions, Love's theory and energy method are used. Computation results show that the fundamental natural frequency comes from different vibration modes while the winding angle varies, the effect of number of axial half waves is stronger than number of circumferential waves on natural frequency of free vibration of anisotropic circular cylindrical shell. The effect of shell's geometrical parameters is also investigated on natural frequencies.
\end{abstract}

\section{Introduction}

Recently because of composite materials' advantages of high intensity and high rigidity, they have been used widely in many fields of engineering, such as aeronautical and aerospace industries. So research of vibration characteristics of composite materials cylindrical shells becomes greatly important. With four different thin shell theories Lam and Loy [1] researched free vibrations of rotating laminated cylindrical shells. Using the energy method, Lee and Kim [2,3] studied the effects of different boundary conditions on the free vibration of rotating composite cylindrical shells with orthogonal stiffeners. Bazhanov [4] investigated free vibration of orthotropic cylindrical shell. But while a certain angle exist between main elastic direction and coordinate axis, how to determine the natural frequencies of cylindrical shell's vibration is not studied. In this paper, such question is investigated with Love's theory and energy method.

\section{Theoretical Formulations}

Fig. 1 shows the considered anisotropic circular cylindrical shell, where $R, l$ and $h$ are the radius, length and thickness of the shell, respectively. The coordinate system $(\alpha, \theta, z)$ is fixed on the middle surface of the shell. The displacements of the shell in the $e_{1}, e_{2}$ and $e_{n}$ direction are denoted by symbols $u, v$ and $w$ respectively.

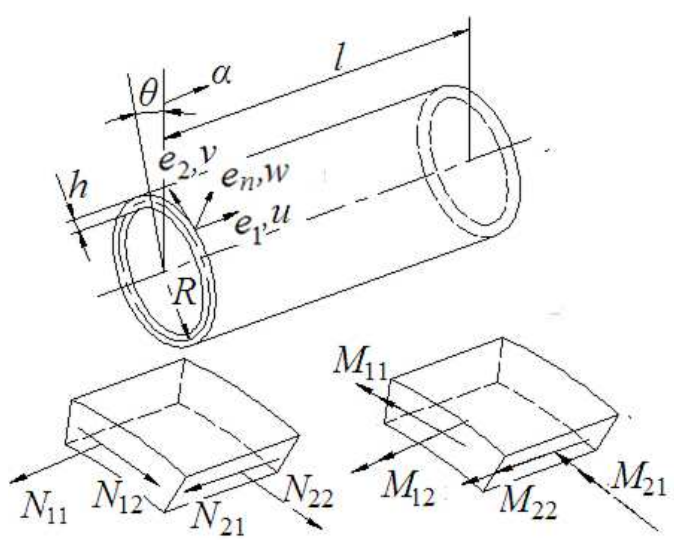

Fig. 1. Coordinate system and geometry of the circular cylindrical shell 
Displacement Functions of Cylindrical Shell. The considered cylindrical shell is with asymmetric boundary conditions. The mathematical expressions for the boundary conditions are given by:

$$
u=v=w=\frac{\partial w}{\partial \alpha}=0, \quad \alpha=0 ; v=w=M_{11}=N_{11}=0, \alpha=l / R .
$$

In this analysis the beam function is used as the axial mode and can be expressed in a general form as [3]:

$$
\psi(\alpha)=\left(\cosh \left(\lambda_{m} R \pi \alpha / l\right)-\cos \left(\lambda_{m} R \pi \alpha / l\right)\right)-\sigma_{m}\left(\sinh \left(\lambda_{m} R \pi \alpha / l\right)-\sin \left(\lambda_{m} R \pi \alpha / l\right)\right) .
$$

Where the coefficient $\sigma_{m}$ is determined by boundary condition and $\lambda_{m}$ does not need an integer as a number depending on the axial wave number.

The admissible displacement functions satisfying the boundary conditions Eq. 1 for freely vibrating anisotropic shells can be written by:

$$
u=A_{m n} \varphi(\alpha) \cos (n \theta) \sin \left(\omega_{m n} t\right) ; v=B_{m n} \psi(\alpha) \sin (n \theta) \sin \left(\omega_{m n} t\right) ; w=C_{m n} \psi(\alpha) \cos (n \theta) \sin \left(\omega_{m n} t\right) .
$$

Where $m$ represents the number of axial half waves, $n$ represents the number of circumferential waves. $\omega_{m n}$ is the angular natural frequency of the free vibration for $(m, n)$ vibration mode, and $A_{m n}, B_{m n}$ and $C_{m n}$ are the amplitudes for each direction for $(m, n)$ vibration mode, respectively. $\varphi(\alpha)=\frac{l}{\lambda_{m} R \pi} \psi^{\prime}(\alpha)$.

Strain and Kinetic Energy of Shell. From the Love's shell theory, the reference surface strains $\varepsilon_{11}, \varepsilon_{22}, \varepsilon_{12}$, the reference surface curvatures $\chi_{11}, \chi_{22}$ and $\chi_{12}$ are defined as follows:

$$
\varepsilon_{11}=\frac{1}{R} \frac{\partial u}{\partial \alpha} ; \varepsilon_{22}=\frac{1}{R}\left(\frac{\partial v}{\partial \theta}+w\right) ; \varepsilon_{12}=\frac{1}{R}\left(\frac{\partial u}{\partial \theta}+\frac{\partial v}{\partial \alpha}\right) ; \chi_{11}=\frac{1}{R^{2}} \frac{\partial^{2} w}{\partial \alpha^{2}} ; \chi_{22}=\frac{1}{R^{2}} \frac{\partial^{2} w}{\partial \theta^{2}} ; \chi_{12}=\frac{2}{R^{2}} \frac{\partial^{2} w}{\partial \alpha \partial \theta}
$$

If there exists a certain winding angle $\varphi$ between main elastic direction and coordinate axis, according to Hook's law the relationship between stresses and strains in the middle surface:

$$
\sigma_{11}=B_{11} \varepsilon_{11}+B_{12} \varepsilon_{22}+B_{16} \varepsilon_{12} ; \sigma_{22}=B_{12} \varepsilon_{11}+B_{22} \varepsilon_{22}+B_{26} \varepsilon_{12} ; \sigma_{12}=B_{16} \varepsilon_{11}+B_{26} \varepsilon_{22}+B_{66} \varepsilon_{12}
$$

Where $B_{11}, B_{12}, B_{16}, B_{12}, B_{22}, B_{26}$ and $B_{66}$ are elastic coefficients. They have the following expressions with the winding angle $\varphi[4]$ :

$$
\left\{\begin{array}{l}
B_{11}=b_{11} \cos ^{4} \varphi+b_{22} \sin ^{4} \varphi+\left(b_{66}+b_{12} / 2\right) \sin ^{2} 2 \varphi ; B_{12}=\left(b_{11}+b_{22}-2 b_{66}\right) \sin ^{2} \varphi \cos ^{2} \varphi+b_{12}\left(\sin ^{4} \varphi+\cos ^{4} \varphi\right) ; \\
B_{16}=\left[\left(b_{22} \sin ^{2} \varphi-b_{11} \cos ^{2} \varphi\right) \sin 2 \varphi\right] / 2+\left[\left(b_{12}+2 b_{66}\right) \sin 4 \varphi\right] / 4 ; B_{22}=b_{11} \sin ^{4} \varphi+b_{22} \cos ^{4} \varphi+\left(b_{66}+b_{12} / 2\right) \sin ^{2} 2 \varphi ; \\
B_{26}=\left[\left(b_{22} \cos ^{2} \varphi-b_{11} \sin ^{2} \varphi\right) \sin 2 \varphi\right] / 2-\left[\left(b_{12}+2 b_{66}\right) \sin 4 \varphi\right] / 4 ; B_{66}=\left(b_{11}+b_{22}-2 b_{12}\right) \sin ^{2} \varphi \cos ^{2} \varphi+b_{66} \cos ^{2} 2 \varphi
\end{array}\right.
$$

Where $b_{11}, b_{22}, b_{12}$ and $b_{66}$ are main elastic modulis for orthotropic cylindrical shell (i.e. $\varphi=0$ ).

The stresses of the surface, the distance between which and the middle surface is $z$, can be written as:

$\sigma_{11}^{\prime}=\sigma_{11}-z\left(B_{11} \chi_{11}+B_{12} \chi_{22}+B_{16} \chi_{12}\right) ; \sigma_{12}^{\prime}=\sigma_{12}-z\left(B_{16} \chi_{11}+B_{26} \chi_{22}+B_{66} \chi_{12}\right) ; \sigma_{22}^{\prime}=\sigma_{22}-z\left(B_{12} \chi_{11}+B_{22} \chi_{22}+B_{26} \chi_{12}\right) .(7)$ 
The internal forces and internal moments could be expressed as followings:

$$
\left\{\begin{array}{l}
N_{11}=\int_{-h / 2}^{h / 2} \sigma_{11}^{\prime} d z ; N_{12}=\int_{-h / 2}^{h / 2} \sigma_{12}^{\prime} d z ; N_{21}=\int_{-h / 2}^{h / 2} \sigma_{12}^{\prime} d z ; N_{22}=\int_{-h / 2}^{h / 2} \sigma_{22}^{\prime} d z ; \\
M_{11}=-\int_{-h / 2}^{h / 2} \sigma_{11}^{\prime} z d z ; M_{12}=-\int_{-h / 2}^{h / 2} \sigma_{12}^{\prime} z d z ; M_{21}=-\int_{-h / 2}^{h / 2} \sigma_{12}^{\prime} z d z ; M_{22}=-\int_{-h / 2}^{h / 2} \sigma_{22}^{\prime} z d z .
\end{array} .\right.
$$

The strain energy for the anisotropic cylindrical shell is expressed as follow:

$U=\frac{1}{2} R^{2} \int_{0}^{2 \pi} \int_{0}^{l / R}\left[N_{11} \varepsilon_{11}+N_{22} \varepsilon_{22}+N_{12} \varepsilon_{12}+M_{11} \chi_{11}+M_{22} \chi_{22}+M_{12} \chi_{12}\right] d \alpha d \theta$

The kinetic energy for the composite cylindrical shell has the following expression:

$T=\frac{1}{2} \rho R^{2} \int_{-h / 2}^{h / 2} \int_{0}^{2 \pi} \int_{0}^{l / R}\left[\left(\frac{\partial u}{\partial t}\right)^{2}+\left(\frac{\partial v}{\partial t}\right)^{2}+\left(\frac{\partial w}{\partial t}\right)^{2}\right] d \alpha d \theta d z$

where $\rho$ represents the density of GFRP.

Frequency Equation of the Anisotropic Cylindrical Shell. The Lagrange equation of motion for the composite cylindrical shell is:

$$
\frac{d}{d t}\left[\frac{\partial T}{\partial \dot{q}_{m n}}\right]-\frac{\partial T}{\partial q_{m n}}+\frac{\partial U}{\partial q_{m n}}=0
$$

where $q_{n n}=\left[\begin{array}{lll}A_{n n} & B_{n n} & C_{n n}\end{array}\right]^{\mathrm{T}}$.

Substituting Eq. 9 and Eq. 10 into Eq. 11 the frequency equations can be deduced and simplified in the following matrix form:

$$
\left[M \omega_{m n}^{2}-K\right] q_{m n}=0
$$

Where $M, K$ are the mass and stiffness matrix, respectively.

Because $q_{m n}$ is not equal to zero, the determinant of the characteristic matrix in Eq. 12 must be zero:

$$
\left|M \omega_{m n}^{2}-K\right|=0
$$

Expanding Eq. 13, a polynomial for the natural frequencies can be obtained as follows:

$$
a_{6} \omega^{6}+a_{4} \omega^{4}+a_{2} \omega^{2}+a_{0}=0
$$

Solving Eq. 14, the frequency values $\omega_{m n}$ (minimum positive value is selected) could be obtained for any certain winding angle $\varphi$.

\section{Computation Results and Analysis}

The geometrical parameters and material properties of the circular cylindrical shell used in the present research are: $b_{1111}=18.3 \mathrm{GPa}, b_{2222}=25.2 \mathrm{GPa}, b_{1122}=2.77 \mathrm{GPa} \quad, b_{1212}=3.5 \mathrm{GPa}$, $\rho=1850 \mathrm{Kg} / \mathrm{m}^{3}, h=1 \mathrm{~mm}, R=0.5 \mathrm{~m}, l=10 \mathrm{~m}$. 
Fig. 2 shows the natural frequencies of 5 vibration modes for the anisotropic cylindrical shell, i.e. the number of axial half waves $m=1$, and the number of circumferential waves $n=1,2,3,4,5$ respectively. Frequency curves $\omega_{12}, \omega_{13}$ intersect. While winding angle $\varphi \leq 0.96\left(\varphi \leq 55^{\circ}\right)$, the fundamental frequency is $\omega_{12}$. While winding angle $\varphi \geq 0.96$, the fundamental frequency is $\omega_{13}$. From Fig. 2, the general behavior of 5 vibration modes is that the frequency values first decrease to a minimum (near $\varphi=0.96$ ) and then increase.

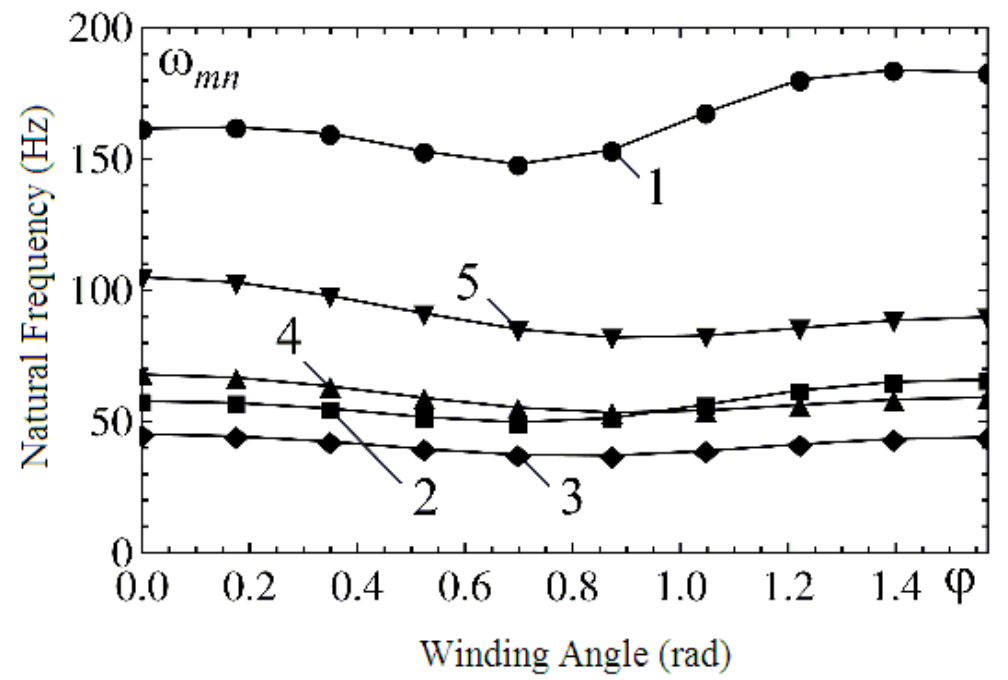

Fig. 2. Variation of the natural frequencies with different winding angle $\varphi(0 \leq \varphi \leq \pi / 2)$

1 -vibration mode $(1,1) ; 2$-vibration mode $(1,2) ; 3$-vibration mode $(1,3)$; 4-vibration mode $(1,4) ; 5$-vibration mode $(1,5)$

Fig. 3 illustrates that the variation tendency of vibration mode $(1,1)$ is smoother than mode $(2,1)$. Compared Fig. 3 with Fig. 2 the effect of value $m$ is stronger than the effect of value $n$ on the natural frequency values of free vibration of circular cylindrical shell.

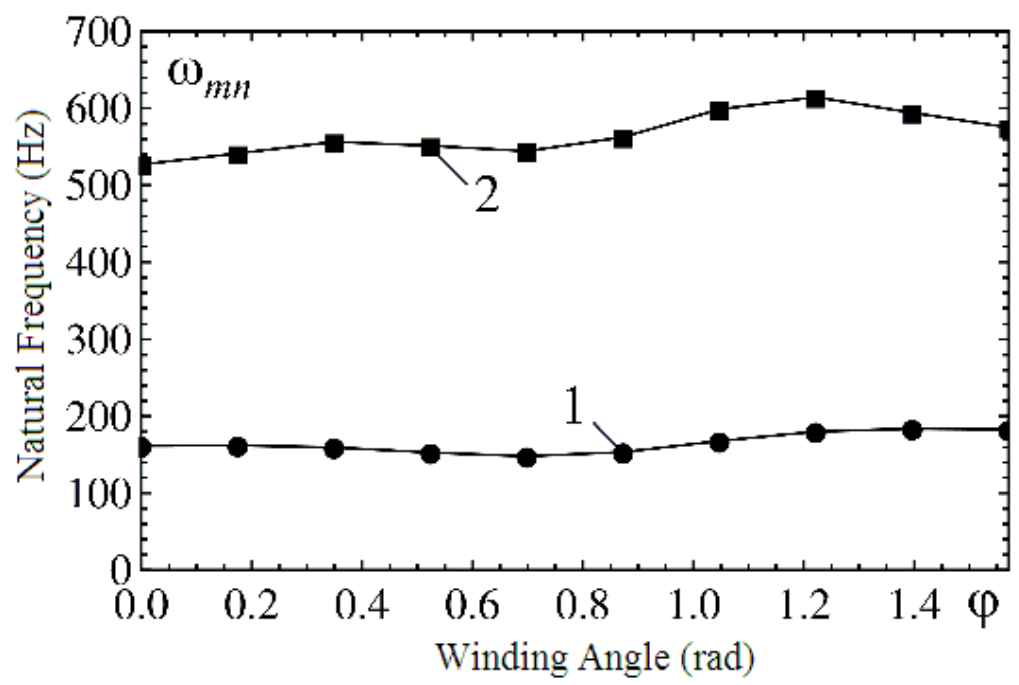

Fig.3. Variation of the natural frequencies with different winding angle $\varphi(0 \leq \varphi \leq \pi / 2)$ 1 -vibration mode $(1,1) ; 2$-vibration mode $(2,1)$

From Fig. 4 it is observed that the effect of variation ratio $h / R$ on the natural frequency is complex. Generally speaking, the bigger the ratio $h / R$ is, the smaller the natural frequency is. And the bigger the ratio $h / R$ is, the smoother the variation of natural frequency is. 


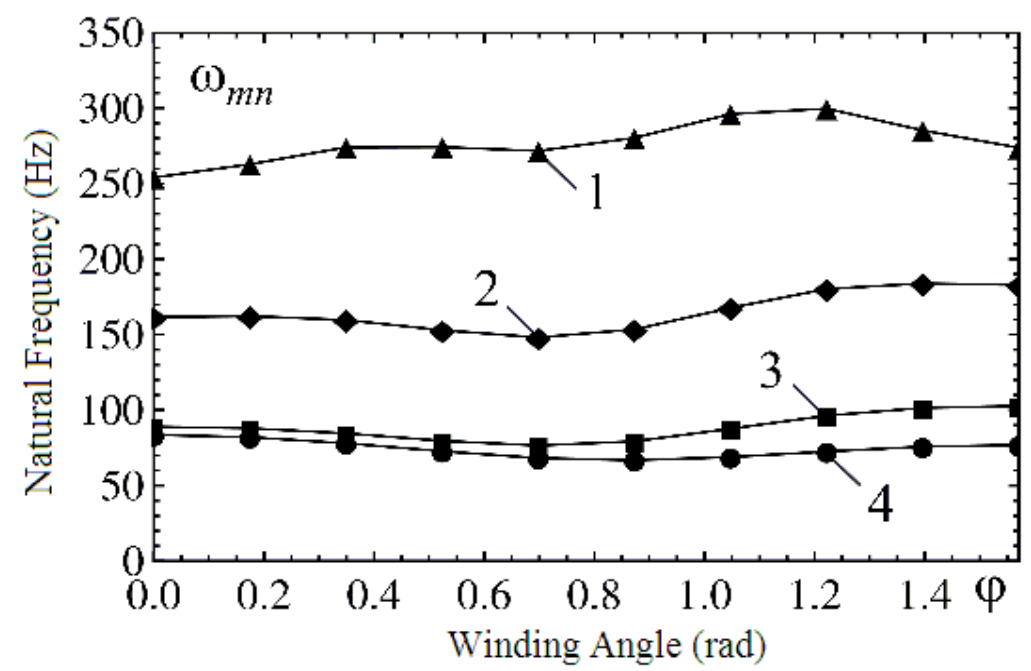

Fig.4. Variation of the natural frequencies with the ratio $h / R$ for different winding angle

$$
\begin{gathered}
\varphi(0 \leq \varphi \leq \pi / 2)(m=n=1) \\
1-h / R=0.001 ; 2-h / R=0.002 ; 3-h / R=0.004 ; 4-h / R=0.01
\end{gathered}
$$

We also can get the effect of variation ratio $R / l$ on the natural frequency. The bigger the ratio $R / l$ is, the bigger the natural frequency is.

\section{Conclusions}

Using Love's shell theory and Energy method, the free vibration of anisotropic circular cylindrical shell with asymmetric boundary conditions has been investigated. The effect of the number of axial half waves is discussed on the mode frequencies as well as the number of circumferential waves. It is also discussed how the shell thickness-to-radius ratio and the shell length-to-radius ratio effect the same vibration mode. During shell design stage this method can be used to determine the natural frequencies. But usually it is not easy to construct displacement functions satisfying boundary conditions.

\section{Acknowledgments.}

This work was supported by Science Technology Item of Heilongjiang Provincial Education Department (No. 12521096).

\section{References}

[1] K. Y. Lam, C. T. Loy, Analysis of rotating laminated cylindrical shells by different thin shell theories Journal of Sound and Vibration. 186(1995), 231-233.

[2] Y. S. Lee, Y. W. Kim, Vibrations analysis of rotating composite cylindrical shells with orthogonal stiffeners, Computers and Structures. 69(1998), 271-281.

[3] Y. S. Lee, Y. W. Kim, Effect of boundary conditions on natural frequencies for rotating composite

[4] V. L. Bazhanov, I. I. Goldenblat, V. A.Kopnov, A. D. Pospelov, A. M Sinykob, Plates and shells of glass fiber-reinforced plastic, High School Press, Moscow, 1970. 